\title{
Bounds on edge colorings with restrictions on the union of color classes
}

\author{
N.R. Aravind and C.R. Subramanian \\ The Institute of Mathematical Sciences, Chennai - 600 113, India. \\ email: $\{$ nraravind,crs\}@imsc.res.in
}

\begin{abstract}
We consider constrained proper edge colorings of the following type: Given a positive integer $j$ and a family $\mathcal{F}$ of connected graphs on 3 or more vertices, we require that the subgraph formed by the union of any $j$ color classes has no copy of any member of $\mathcal{F}$. This generalizes some well-known types of colorings such as acyclic edge colorings, distance-2 edge colorings, low treewidth edge colorings, etc.

For such a generalization of restricted colorings, we obtain an upper bound of $O\left(d^{\max (\theta, 1)}\right)$ on the minimum number of colors used in such a coloring. Here, $d$ refers to the maximum degree of the graph and $\theta$ is a parameter defined by $\theta=\theta(j, \mathcal{F})=\max _{H \in \mathcal{F}} \frac{(|V(H)|-2)}{(|E(H)|-j)}$. Our proof is based on probabilistic arguments. In particular, we obtain $O(d)$ upper bounds for proper edge colorings with various interesting restrictions placed on the union of color classes. For example, we obtain $O(d)$ upper bounds on edge colorings with restrictions such as $(i)$ the union of any 3 color classes should be an outerplanar graph, (ii) the union of any 4 color classes should have treewidth at most 2, (iii) the union of any 5 color classes should be planar, (iv) the union of any 16 color classes should be 5-degenerate, etc.
\end{abstract}

We also consider generalizations where we require simultaneously for several pairs $\left(j_{i}, \mathcal{F}_{i}\right)(i=1, \ldots, s)$ that the union of any $j_{i}$ color classes has no copy of any member of $\mathcal{F}_{i}$ and obtain upper bounds on the corresponding chromatic indices. As a corollary, we obtain that each of the four restrictions above can be satisfied simultaneously using $O(d)$ colors. 
Some ways of improving the bounds are sketched. Also, if we drop the requirement that the edge coloring be proper, then an $O\left(d^{\theta}\right)$ upper bound on the chromatic index is established. Further, the stated upper bounds are also bounds for the list analogues of these edge colorings.

Proposed running head : Bounds on restricted edge colorings.

Key words : edge colorings, acyclic edge colorings, treewidth, probabilistic arguments, maximum degree.

AMS subject classification : $05 \mathrm{C} 15,05 \mathrm{D} 40$.

\section{Introduction}

All graphs considered here are simple and undirected. A proper edge coloring is a labeling of the edges of a graph such that touching edges (i.e. edges sharing a common endpoint) do not get the same color. The minimum number of colors used in a proper edge coloring of a graph $G$ is called the chromatic index and is denoted by $\chi^{\prime}(G)$. This is a well-studied parameter and it is known from a celebrated theorem of Vizing [?] that $\chi^{\prime}(G)$ is always at most $\Delta+1$ where $\Delta$ denotes the maximum degree of a vertex in $G$.

Several variants of edge colorings have been studied by imposing additional restrictions on the colorings. An interesting example is the acyclic edge coloring which is a proper coloring of the edges of a graph such that there are no bichromatic cycles in the coloring, equivalently, the union of any two color classes must form a forest. Alon, McDiarmid and Reed ([1]) showed that if $G$ has maximum degree $d$, then the acyclic chromatic index is $O(d)$. A distance-2 edge coloring or a strong edge coloring is a proper edge coloring in which edges adjacent to a common edge must also get distinct colors and it is always possible to obtain such a coloring using $O\left(d^{2}\right)$ colors.

Recently, a general notion of a restricted vertex coloring in which we place some restrictions on the union of color classes was considered by Nesetril and Ossona de Mendez in [7], and in [9], the present authors obtained bounds for these types of colorings in terms of the maximum degree of the graph. In this paper, we consider the edge analogues of such restricted colorings and obtain similar upper bounds. 
In the case of acyclic coloring, it turns out (see [1]) that any acyclic vertex coloring requires $\Omega\left(d^{4 / 3} /(\log d)^{1 / 3}\right)$ colors for some graphs while, as mentioned before, an acyclic edge coloring is always possible using only $O(d)$ colors. We might expect that restricted edge colorings of such type, in general, require fewer number of colors than their vertex analogues and in this paper we show that this is indeed true for several types of edge colorings. In fact, we show that for several such edge colorings (like those mentioned in the abstract), the upper bound is simply $O(d)$.

First, we formally define a general notion of a restricted edge coloring.

Definition 1.1 Let $\mathcal{F}$ be a family of connected graphs and let $j$ be a positive integer such that $j<\min _{H \in \mathcal{F}}(|E(H)|)$. We define a $(j, \mathcal{F})$ edge coloring of a graph $G$ to be a proper coloring of $E(G)$ such that the subgraph of $G$ formed by the union of any $j$ color classes does not contain an isomorphic copy of $H$ as a subgraph (not necessarily induced), for each $H \in F$. We denote by $\chi_{j, F}^{\prime}(G)$ the minimum number of colors sufficient for a $(j, \mathcal{F})$ edge coloring of $G$.

Remark: We require $j<|E(H)|$ for each $H \in F$ because otherwise if $G$ contains a copy of $H$ such that $j \geq|E(H)|$, no proper coloring of $E(G)$ would be a $(j, F)$ edge coloring. Also if $j<|E(H)|$ for each $H \in F$, we are guaranteed of at least one $(j, \mathcal{F})$ edge coloring, namely the trivial coloring (in which each edge gets a distinct color).

Notation: For a positive integer $j$ and a family $\mathcal{F}$ of graphs such that $j<E(H)$ for each $H \in \mathcal{F}$, we define and use $\theta(j, \mathcal{F})$ to denote the expression (SUP stands for the supremum) below:

$$
S U P_{H \in \mathcal{F}} \frac{(|V(H)|-2)}{(|E(H)|-j)}
$$

The main result of this paper is the following theorem which provides upper bounds on the optimal number of colors used in such a coloring.

Theorem 1.1 Let $\mathcal{F}$ be a family of connected graphs and let $j$ be a positive integer such that $j<\min _{H \in \mathcal{F}}|E(H)|$. Let $\theta=\theta(j, \mathcal{F})$. Then, there exists a constant $C=C(j, \mathcal{F})$ such that for any graph $G$ of maximum degree $d$, $\chi_{j, \mathcal{F}}^{\prime}(G) \leq C d^{\max (\theta, 1)}$. 
As mentioned before, the acyclic chromatic index of any graph of maximum degree $d$ is at most $O(d)$. This naturally leads to the general question of determining those $(j, \mathcal{F})$ pairs for which $\chi_{j, \mathcal{F}}^{\prime}(G)=O(d)$. The following corollary of the previous theorem provides an answer to this question.

Corollary 1.2 Let $\mathcal{F}$ be a family of connected graphs and let $D=D(\mathcal{F})=$ $\min _{H \in \mathcal{F}}|E(H)-| V(H) \mid$. Let $j$ be any positive integer such that $j<\min _{H \in \mathcal{F}}|E(H)|$ and $j \leq D+2$. Then there exists a constant $C=C(\mathcal{F})$ such that for any graph $G$ of maximum degree $d, \chi_{j, \mathcal{F}}^{\prime}(G) \leq C d$.

Proof Follows from Theorem 1.1 after applying the easy to verify observation that $\theta \leq 1$ if and only if $j \leq D+2$.

Outline : In Section 2, we state and prove an explicit version (Proposition 2.2) of Theorem 1.1. Also, at the end of this section, we present an analogue of this result for free $(j, \mathcal{F})$ edge colorings wherein we drop the insistence on properness of the edge coloring. In Section 3, we apply this proposition to obtain bounds for several interesting restrictions on color classes. In Section 4, we extend the results to edge colorings where we require several families forbidden simultaneously. In Section 5, we discuss some ways of improving the upper bounds further for some graph classes. In Section 6, we discuss some improvements for free colorings and also discuss bounds on list analogues of $(j, \mathcal{F})$ edge colorings. Finally, in Section 7 , we conclude with some remarks and open problems.

\section{Proof of results}

The proof of Theorem 1.1 is based on probabilistic arguments and is based on a non-trivial application of Lovász Local Lemma. We use the following non-symmetric form of Lovász Local Lemma (see $[2, ?])$.

Lemma 2.1 (see [?]) Let $\mathcal{A}=\left\{A_{1}, A_{2}, \ldots, A_{n}\right\}$ be a family of events in an arbitrary probability space such that each event $A_{i}$ is mutually independent of $\mathcal{A} \backslash\left(\left\{A_{i}\right\} \cup \mathcal{D}_{i}\right)$ for some $\mathcal{D}_{i} \subset \mathcal{A}$.

Then if there are reals $0<y_{i}<1$ such that for all $i$,

$$
\operatorname{Pr}\left(A_{i}\right) \leq y_{i} \prod_{\mathcal{A}_{j} \in \mathcal{D}_{i}}\left(1-y_{j}\right)
$$

then

$$
\operatorname{Pr}\left(\cap\left(\overline{A_{i}}\right)\right) \geq \prod_{i=1}^{n}\left(1-y_{i}\right)>0
$$


so that with positive probability no event $A_{i}$ occurs.

We prove the following explicit version of Theorem 1.1, wherein we have not attempted to optimize the constant $C(j, \mathcal{F})$.

Proposition 2.2 Let $\mathcal{F}$ be a family of connected graphs and let $j$ be a positive integer as in Theorem 1.1. Let

$$
\begin{gathered}
\theta=\theta(j, \mathcal{F})=S U P_{H \in \mathcal{F}} \frac{(|V(H)|-2)}{(|E(H)|-j)}, \\
D=D(\mathcal{F})=\min _{H \in \mathcal{F}}(|E(H)-| V(H) \mid), \\
C=C(j, \mathcal{F})=200 \cdot 2^{6 j+6 D} \cdot(3 j)^{2 j} .
\end{gathered}
$$

Then, for any graph $G$ of maximum degree $d, \chi_{j, \mathcal{F}}^{\prime}(G) \leq(C d)^{\max (\theta, 1)}$.

\section{Proof of Proposition 2.2:}

Let $G=(V, E)$ be the given graph. Without loss of generality, we assume that $j \geq 2$. When $j=1$, any $(j, \mathcal{F})$ coloring is the same as a proper edge coloring of $G$ which always exists with $d+1$ colors (by Vizing's Theorem, see [4], pages-277-279). Henceforth, we assume that $j \geq 2$.

Put $x=\left\lceil(C d)^{\max (\theta, 1)}\right\rceil$ where $C=200 \cdot 2^{6 j+6 D} \cdot(3 j)^{2 j}$.

Let $f: E \rightarrow\{1,2, \ldots, x\}$ be a random edge coloring of $G$, where for each edge $e \in E$ independently, the color $f(e) \in\{1,2, \ldots, x\}$ is chosen uniformly at random. It suffices to prove that with positive probability, $f$ is a $(j, \mathcal{F})$ edge coloring of $G$. To this end, we define a family of bad events whose absence implies that the random coloring is a $(j, \mathcal{F})$ edge coloring and use the Lovász Local Lemma to show that with positive probability none of these events occur. The events we consider are of the following two types.

Type I: For each pair of touching edges $e_{1}=(u, v)$ and $e_{2}=(u, w)$, let $A_{e_{1}, e_{2}}$ be the event that $f\left(e_{1}\right)=f\left(e_{2}\right)$.

We define $\alpha=\frac{1}{\theta}$. The definition of the Type II event depends on whether $\alpha<1$ or $\alpha \geq 1$. 
le Case $\alpha<1$ :

Type II: For each connected subgraph $L$ of $G$ such that $|V(L)| \geq 3$ and $|E(L)|=\max \{|V(L)|-1,\lceil\alpha(|V(L)|-2)+j\rceil\}$, let $B_{L}$ be the event that the edges in $L$ are colored using at most $j$ colors in the coloring by $f$.

Note that for each $H \in \mathcal{F}$, we have $|E(H)| \geq|V(H)|-1$ and $|E(H)| \geq$ $\lceil\alpha(|V(H)|-2)+j\rceil$ and hence the absence of type II events in this case ensures that the union of any $j$ color classes cannot have a copy of any member of $\mathcal{F}$.

\section{Case $\alpha \geq 1$ :}

Type II: For each connected subgraph $L$ of $G$ such that $|V(L)| \geq 3$ and $|E(L)|=|V(L)|+D$, let $B_{L}$ be the event that the edges in $L$ are colored using at most $j$ colors in the coloring by $f$. Since, by assumption, $j \geq 2$, we have $D \geq 0$. Also, for each $H \in \mathcal{F}$, we have $|E(H)| \geq|V(H)|+D$ and thus the absence of type II events in this case ensures that the union of any $j$ color classes cannot have a copy of any member of $\mathcal{F}$.

Thus we see that if none of the events of the two types above occur, then $f$ is a $(j, \mathcal{F})$ edge coloring.

It remains to show that with positive probability none of these events happen. To prove this, we apply the Lovász Local Lemma. Note that any event of either of the two types is mutually independent of all events that do not share an edge in common with the given event.

We need to estimate the number of events of each type possibly influencing any given event. This estimate follows from the following two simple lemmas.

Lemma 2.3 Let $e=(u, v)$ be an arbitrary edge of the graph $G=(V, E)$. Then the following two statements hold.

(i) e touches at most $2 d$ edges in $G$.

(ii) e belongs to at most $2 k^{2 j+2 D+1} 4^{k} d^{k-2}$ subgraphs of $V(G)$ on $k$ vertices which are as in a Type II event.

Proof Part (i) follows from the fact that $\Delta(G)=d$.

Part (ii) can be seen as follows: If $\alpha<1$, let $\mathcal{G}(e, k)$ be the set of connected subgraphs (containing $e$ ) in $G$ on $k$ vertices and having $\max \{k-$ $1,\lceil\alpha(k-2)+j\rceil\}$ edges. If $\alpha \geq 1$, let $\mathcal{G}(e, k)$ be the set of connected subgraphs 
(containing $e$ ) in $G$ on $k$ vertices and having $k+D$ edges. Let $\mathcal{T}(e, k)$ be the set of $k$-vertex trees in $G$ containing $e$ with some arbitrary linear order imposed on them.

If $\alpha<1$, each tree in $\mathcal{T}(e, k)$ is a subgraph of at most

$$
\left(\begin{array}{c}
\left(\begin{array}{l}
k \\
2
\end{array}\right) \\
\max \{0,\lceil\alpha(k-2)+j\rceil-(k-1)\}
\end{array}\right) \leq k^{2 j-2}
$$

connected subgraphs in $\mathcal{G}(e, k)$ on the same set of vertices. If $\alpha \geq 1$, each tree in $\mathcal{T}(e, k)$ is a subgraph of at most $\left(\begin{array}{c}k \\ 2 \\ D+1\end{array}\right) \leq k^{2 D+2}$ connected subgraphs in $\mathcal{G}(e, k)$ on the same set of vertices. Each connected subgraph $H$ in $\mathcal{G}(e, k)$ has at least one tree in $\mathcal{T}(e, k)$ the smallest (with respect to the assumed linear ordering) of which is identified with $H$. Thus $|\mathcal{G}(e, k)| \leq k^{2 j+2 D}|\mathcal{T}(e, k)|$, irrespective of whether $\alpha<1$ or $\alpha \geq 1$.

We now find an upper bound for $|\mathcal{T}(e, k)|$. Since there are at most $4^{k}$ unlabeled trees on $k$ vertices (see Chapter 8 of [6]), there are at most $4^{k}$ choices for choosing the unlabeled structure of a tree in $\mathcal{T}(e, k)$. Once this unlabeled structure is fixed, we now have to embed this unlabeled tree in $G$. The number of ways of identifying edge $e$ with an edge in the unlabeled tree is at most $2(k-1)<2 k$. Now the remaining vertices in the unlabeled tree can be embedded in at most $d^{k-2}$ ways. To see this, we observe that there are $d$ choices for each neighbor of $v$ in the chosen unlabeled tree. Once these are fixed, the number of choices for a neighbor of each first neighbor is again $d$. Repeating this process, we can see that the number of choices for embedding all the vertices (other than $u, v$ ) is at most $d^{k-2}$. This proves (ii).

Lemma 2.4 For $\{i, j\} \in\{I, I I\}$ the $(i, j)$-th entry of the table given below is an upper bound on the number of events of type $j$ which can possibly influence an event of type $i$.

\begin{tabular}{|c|c|c|}
\hline & $I$ & $I I\left(B_{L^{\prime}}\right)$ \\
\hline$I$ & $4 d$ & $4 l^{2 j+2 D+1} 4^{l} d^{l-2}$ \\
\hline$I I\left(B_{L}\right)$ & $2 m d$ & $2 m l^{2 j+2 D+1} 4^{l} d^{l-2}$ \\
\hline
\end{tabular}

Here, $m$ is the number of edges in $L$ and $l$ is the number of vertices in $L^{\prime}$. The lemma follows from Lemma 2.3 and the fact that any event is mutually 
independent of all other events which do not share any edge with the given event. We now estimate the probability of occurrence of each type of event.

Fact 2.5 (i) For each type I event $A, \operatorname{Pr}(A)=\frac{1}{x}$.

(ii) For each type II event $B_{L}, \operatorname{Pr}\left(B_{L}\right) \leq \frac{j^{m}}{x^{m-j}}$, where $m=|E(L)|$.

The number of ways in which $m$ edges can be colored using at most $j$ colors from $\{1,2, \ldots, x\}$ is at most $\left(\begin{array}{l}x \\ j\end{array}\right) j^{m} \leq x^{j} j^{m}$. This proves (ii).

We now define the constants $y_{i}$ to enable us to apply the Local Lemma. For an event $A$ of type $I$, we define $y_{A}=\frac{9}{x}$. For an event $B_{L}$ of type $I I$, we define $y_{B_{L}}=\frac{(3 j)^{m}}{x^{m-j}}$, where $m=|E(L)|$.

If $\alpha<1,|E(L)|-j \geq \alpha(|V(L)|-2)$ for each forbidden $j$-colored graph $L$ and using $x>3 j$, we note that $y_{B_{L}} \leq \frac{(3 j)^{j+\alpha(k-2)}}{x^{\alpha(k-2)}}$ where $k=|V(L)|$.

If $\alpha \geq 1$, then $|E(L)|-j \geq|V(L)|-2$ for each forbidden $j$-colored graph $L$ and hence $y_{B_{L}}=\frac{(3 j)^{k+D}}{x^{k+D-j}} \leq \frac{(3 j)^{k+j-2+D-j+2}}{x^{k-2+D-j+2}} \leq \frac{(3 j)^{k+j-2}}{x^{k-2}}$, where $k=|V(L)|$. Here we used $x>3 j$ and also the fact that $D \geq j-2$ whenever $\alpha \geq 1$.

In either case, by substituting $x=(C d)^{\max (\theta, 1)}$, we find that $y_{B_{L}} \leq$ $\frac{(3 j)^{k+j-2}}{(C d)^{k-2}}$ and hence $\left(1-y_{B_{L}}\right) \geq 1-\frac{(3 j)^{j+k-2}}{(C d)^{k-2}}$.

By Lemma 2.1, Lemma 2.4 and Fact 2.5, it thus suffices to verify the following two inequalities.

$$
\begin{gathered}
\frac{1}{x} \leq \frac{9}{x}\left(1-\frac{9}{x}\right)^{4 d} \prod_{l \geq 3}\left(1-y_{B_{L}^{\prime}}\right)^{42^{2 j+2 D+1} 4^{l} d^{l-2}}-(\mathbf{1}) \\
\frac{j^{m}}{x^{m-j}} \leq \frac{(3 j)^{m}}{x^{m-j}}\left(1-\frac{9}{x}\right)^{2 m d} \prod_{l \geq 3}\left(1-y_{B_{L}^{\prime}}\right)^{2 m l^{2 j+2 D+1} 4^{l} d^{l-2}}, \quad \forall m \geq 3-
\end{gathered}
$$

We see that (2) is equivalent to (1). Thus it is sufficient to prove (1). 
In (1), we substitute $x=(C d)^{\max (\theta, 1)}$ where $C=200 \cdot(2)^{6 j+6 D} \cdot(3 j)^{2 j}$ and using the known fact that $\left(1-\frac{1}{z}\right)^{z} \geq 1 / 4$ for all $z \geq 2$, as well as the fact that $\left(1-y_{B_{L^{\prime}}}\right) \geq 1-\frac{(3 j)^{j+l-2}}{(C d)^{(l-2)}}$ we see that it is sufficient to prove:

$$
\frac{1}{9} \leq 4^{-\frac{36 d}{x}} 4^{-S}
$$

where

$$
S=\sum_{l \geq 3} \frac{(3 j)^{j+l-2} \cdot 4^{l+1} \cdot l^{2 j+2 D+1}}{200^{l-2} \cdot 2^{(6 j+6 D)(l-2)} \cdot(3 j)^{(2 j)(l-2)}}
$$

Using the fact that

$$
j+l-2 \leq 2 j(l-2), \quad \forall j \geq 2, l \geq 3
$$

and also the fact that

$$
l^{2 j+2 D+1}<2^{(2 j+2 D) l} \leq 2^{(6 j+6 D)(l-2)}, \quad \forall j \geq 2, l \geq 3, D \geq-1,
$$

we get

$$
S \leq \sum_{l \geq 3} \frac{4^{l+1}}{200^{l-2}}=\frac{64}{49}<\frac{4}{3}
$$

We thus find that it is sufficient to prove:

$$
\frac{1}{9} \leq 4^{-\frac{36 d}{x}} 4^{-\frac{4}{3}}
$$

Since $x \geq 216 d$, the above inequality is true.

Thus by Lovász Local Lemma, with positive probability, none of the bad events occur and hence a $(j, \mathcal{F})$ edge coloring exists using $O\left(d^{\max (\theta, 1)}\right)$ colors. This completes the proof of Proposition 2.2 and hence of Theorem 1.1.

\subsection{Free $(j, \mathcal{F})$ edge colorings}

Suppose, in Definition 1.1, we do not explicitly insist that the edge coloring be proper. We call such a coloring a free (from having to be proper) $(j, \mathcal{F})$ edge coloring. We use the notation $f \chi_{j, \mathcal{F}}^{\prime}(G)$ to denote the corresponding free chromatic index. It follows that there is an analogue of Proposition 2.2 corresponding to free $(j, \mathcal{F})$ edge colorings also. It is given below. 
Proposition 2.6 Let $\mathcal{F}, j, \theta=\theta(j, \mathcal{F}), D=D(\mathcal{F}), C=C(j, \mathcal{F})$ be all the same as defined in Proposition 2.2 except that $C(1, \mathcal{F})$ is redefined to be $7200 \cdot 2^{6+6 D}$. Then, for any graph $G$ of maximum degree $d$, the free $(j, \mathcal{F})$ chromatic index is bounded as $f \chi_{j, \mathcal{F}}^{\prime}(G) \leq(C d)^{\theta}$.

By setting $j=1$ and $\mathcal{F}=\left\{K_{t, t}\right\}$, we see that $\theta(j, \mathcal{F})=\frac{2 t-2}{t^{2}-1}=\frac{2}{t+1}$ and hence $E\left(K_{n}\right)$ can be partitioned into $O\left(n^{2 /(t+1)}\right)$ parts so that each part has no copy of $K_{t, t}$. This strengthens a well-known fact in extremal graph theory (see [?]), namely, that there is a $K_{t, t}$-free graph on $n$ vertices having $\Omega\left(n^{2-2 /(t+1)}\right)$ edges. In particular, it follows that there are $C_{4}$-free graphs on $n$ vertices having at least $\Omega\left(n^{4 / 3}\right)$ edges. Similarly, it follows that there is a coloring of $E\left(K_{n}\right)$ using $O\left(n^{1 / 2}\right)$ colors so that each color class is trianglefree.

\section{Consequences}

We now apply Theorem 1.1 and Proposition 2.6 to some interesting families of graphs to obtain the results in the following table. Throughout, we assume that $\Delta(G)=d$. 


\begin{tabular}{|c|c|c|c|c|}
\hline $\begin{array}{l}\text { Restriction on } \\
\text { the union of } \\
\text { color classes }\end{array}$ & $\mathrm{j}$ & $\mathcal{F}$ & $\theta(j, \mathcal{F})$ & $\begin{array}{c}\text { Bound on } \\
\chi_{j, \mathcal{F}}^{\prime}(G)\end{array}$ \\
\hline Planar & 5 & Subdivisions of $K_{3,3}$ and $K_{5}$ & 1 & $O(d)$ \\
\hline$" \eta$ & 6 & $" \%$ & $4 / 3$ & $O\left(d^{4 / 3}\right)$ \\
\hline$" "$ & 7 & $" \%$ & 2 & $O\left(d^{2}\right)$ \\
\hline$" n$ & 8 & $" n$ & 4 & $O\left(d^{4}\right)$ \\
\hline Outerplanar & 3 & Subdivisions of $K_{4}$ and $K_{2,3}$ & 1 & $O(d)$ \\
\hline$" \prime$ & 4 & $" n$ & $3 / 2$ & $O\left(d^{3 / 2}\right)$ \\
\hline$" n$ & 5 & $" n$ & 3 & $O\left(d^{3}\right)$ \\
\hline $\begin{array}{l}\text { Treewidth } \\
\text { at most } 2\end{array}$ & 4 & Subdivisions of $K_{4}$ & 1 & $O(d)$ \\
\hline$" \%$ & 5 & $" n$ & 2 & $O\left(d^{2}\right)$ \\
\hline $\begin{array}{l}\text { Treewidth } \\
\text { at most } k \\
\text { for } k \geq 2\end{array}$ & $k+2$ & $\begin{array}{l}\text { Edge minimal graphs of } \\
\text { treewidth more than } k\end{array}$ & 1 & $O(d)$ \\
\hline $\begin{array}{l}k \text {-degenerate } \\
\text { graphs }\end{array}$ & $\frac{k^{2}+k+2}{2}$ & $\begin{array}{l}\text { Edge minimal graphs that } \\
\text { are non- } k \text {-degenerate }\end{array}$ & 1 & $O(d)$ \\
\hline $\begin{array}{l}k \text {-colorable } \\
\text { graphs }\end{array}$ & $\frac{k^{2}-k+2}{2}$ & $\begin{array}{l}\text { Edge-critical }(k+1)- \\
\text { chromatic graphs }\end{array}$ & 1 & $O(d)$ \\
\hline $\begin{array}{l}\text { Genus } \\
\text { at most } g\end{array}$ & $2 g+3$ & $\begin{array}{l}\text { Edge minimal graphs of } \\
\text { genus more than } g\end{array}$ & 1 & $O(d)$ \\
\hline
\end{tabular}

\section{Justification for some entries :}

1. Planarity restriction:

Note that any subdivision of $K_{5}$ is a graph on $5+k$ vertices and $10+k$ edges for some $k \geq 0$. Similarly, any subdivision of $K_{3,3}$ is a graph on $6+l$ vertices and $9+l$ edges for some $l \geq 0$. Hence

$$
\theta(j, \mathcal{F})=S U P_{k, l \geq 0}\left\{\frac{3+k}{10-j+k}, \frac{4+l}{9-j+l}\right\} .
$$

This value is atmost 1 if $j \leq 5$ and is $4 / 3$ for $j=6$ and is 2 for $j=7$ and is 4 for $j=8$. This proves the entries in the table.

2. Outerplanarity restriction :

Note that any subdivision of $K_{4}$ is a graph on $4+k$ vertices and $6+k$ edges for some $k \geq 0$. Similarly, any subdivision of $K_{2,3}$ is a graph on 
$5+l$ vertices and $6+l$ edges for some $l \geq 0$. Hence

$$
\theta(j, \mathcal{F})=S U P_{k, l \geq 0}\left\{\frac{2+k}{6-j+k}, \frac{3+l}{6-j+l}\right\} .
$$

This value is atmost 1 if $j \leq 3$ and is $3 / 2$ for $j=4$ and is 3 for $j=5$. This proves the entries in the table.

3. $k$-degeneracy restriction :

Any connected minimal (with respect to edge deletion) graph of degeneracy $k+1$ is a graph on $v$ vertices for some $v \geq k+2$ and has minimum degree $k+1$ and hence has at least $v(k+1) / 2$ edges. Thus, $D \geq(k+2)(k-1) / 2$ amd hence for $j \leq \frac{(k+2)(k-1)}{2}+2=\frac{k^{2}+k+2}{2}$, we can apply Corollary 1.2 to deduce that $O(d)$ colors suffice.

4. $k$-colorability restriction :

Any connected minimal (with respect to edge deletion) graph of chromatic number $k+1$ is a graph on $v$ vertices for some $v \geq k+1$ and has minimum degree at least $k$ and hence has at least $v k / 2$ edges. Thus, $D \geq(k+1)(k-2) / 2$ and hence for $j \leq \frac{(k+1)(k-2)}{2}+2=\frac{k^{2}-k+2}{2}$, we can apply Corollary 1.2 to deduce that $O(d)$ colors suffice.

5. treewidth at most $k$ :

It can be shown by a simple inductive argument that any connected graph on $v$ vertices and having treewidth more than $k$ contains at least $v+k$ edges provided $k \geq 2$. This shows that for $j \leq k+2, \theta(\mathcal{F}) \leq 1$.

6. Genus at most $g$ :

By Euler's polyhedral formula, the number of edges in a graph of genus at least $g+1$ and having $v$ vertices is at least $v+2 g+1$. Thus $D(\mathcal{F})=\min _{H \in \mathcal{F}}(|E(H)-| V(H) \mid) \geq 2 g+1$. Hence, by Corollary 1.2, for $j \leq 2 g+3, O(d)$ colors suffice.

\section{Extensions to colorings with several families for- bidden simultaneously}

We can also extend our results to more restricted edge colorings where we require simultaneously for several pairs $\left(j_{i}, \mathcal{F}_{i}\right)(i=1, \ldots, s)$ that the union of any $j_{i}$ color classes has no copy of any member of $\mathcal{F}_{i}$. The vertex versions of such colorings were considered by Nešetruil and Ossona de Mendez in [7] 
for families of $H$-minor-free graphs. A slightly relaxed notion (where we don't insist on properness) was studied by DeVos, et. al. in [3] for families of $\mathrm{H}$-minor-free graphs. However, we obtain bounds which work for any arbitrary graph $G$. We first formally define these colorings.

Definition 4.1 Let $\mathcal{P}=\left\{\left(j_{1}, \mathcal{F}_{1}\right), \ldots,\left(j_{s}, \mathcal{F}_{s}\right)\right\}$ be a set of $s \geq 1$ pairs such that for each $i \leq s, j_{i}$ is a positive integer and $\mathcal{F}_{i}$ is a family of connected graphs such that $j_{i}<|E(H)|$ for each $H \in \mathcal{F}_{i}$. We define a $\mathcal{P}$-edge coloring to be a proper edge coloring of $G$ so that, for each $i \leq s$, the union of any $j_{i}$ color classes does not contain an isomorphic copy of $H$ as a subgraph, for each $H \in \mathcal{F}_{i}$. We denote by $\chi_{\mathcal{P}}^{\prime}(G)$ the minimum number of colors sufficient for a $\mathcal{P}$-edge coloring of $G$.

Note : Similarly, one can define the free version (without explicitly insisting on properness) of a $\mathcal{P}$-edge coloring and denote the corresponding chromatic index by $f \chi_{\mathcal{P}}^{\prime}(G)$.

We now present the main result of this section. We skip the proof of the following theorem as it is based on an application of the Local Lemma and is similar to the proofs of Theorem 1.1 and Proposition 2.6.

Theorem 4.1 Let $\mathcal{P}=\left\{\left(j_{1}, \mathcal{F}_{s}\right), \ldots,\left(j_{s}, \mathcal{F}_{s}\right)\right\}$ be a set of $s \geq 1$ pairs such that for each $i \leq s, j_{i}$ is a positive integer and $\mathcal{F}_{i}$ is a family of connected graphs such that for each $j_{i}<|E(H)|$ for each $H \in \mathcal{F}_{i}$. Define

$$
\begin{gathered}
\theta_{i}=\theta\left(j_{i}, \mathcal{F}_{i}\right)=S U P_{H \in \mathcal{F}_{i}} \frac{(|V(H)|-2)}{\left(|E(H)|-j_{i}\right)}, \forall i \leq s, \\
D_{i}=D\left(\mathcal{F}_{i}\right)=\min _{H \in \mathcal{F}_{i}}(|E(H)-| V(H) \mid), \forall i \leq s, \\
C_{i}=C\left(j_{i}, \mathcal{F}_{i}\right)=200 s \cdot 2^{6 j_{i}+6 D_{i}} \cdot\left(3 j_{i}\right)^{2 j_{i}}, \forall i \leq s, \\
\theta=\max _{i \leq s} \theta_{i}, \quad C=\max _{i \leq s} C_{i} .
\end{gathered}
$$

Then, for any graph $G$ of maximum degree $d, \chi_{\mathcal{P}}^{\prime}(G) \leq(C d)^{\max (\theta, 1)}$. Also, in the case of free $\mathcal{P}$-colorings, we have $f \chi_{\mathcal{P}}^{\prime}(G) \leq(C d)^{\theta}$ with $C_{i}$ being redefined as $C_{i}=7200 \mathrm{~s} \cdot 2^{6\left(D_{i}+1\right)}$ if $j_{i}=1$. 
By setting $\mathcal{P}_{s}=\left\{\left(1, \mathcal{F}_{1}\right), \ldots,\left(s, \mathcal{F}_{s}\right)\right\}$ where $\mathcal{F}_{i}$ is the set of all $i$ colorable (usual edge coloring) graphs of treewidth $i+1$, for each $i \leq s$, we get upper bounds on the the type of edge colorings studied by DeVos, et. al. in [3].

Corollary 4.2 For $s \geq 1$, let $\chi_{\mathcal{P}_{s}}^{\prime}(G)$ denote the minimum number of colors sufficient to obtain a proper edge coloring of $G$ so that the union of any $j \leq s$ color classes forms a subgraph of treewidth at most $j$. Then, there exists a constant $C=C(s)$ such that for any graph of maximum degree $d$, $\chi_{\mathcal{P}_{s}}^{\prime}(G) \leq C \dot{d}$.

Remark : It is essential that $s$ (the number of distinct $j$ 's) of Theorem 4.1 is finite. If we allow $s$ to be infinite, then it is possible that the corresponding chromatic number number may not be bounded by a function of maximum degree $d$ alone. For example, if $\mathcal{P}=\left\{\left(k-1,\left\{P_{k}\right\}\right): k \geq 2\right\}\left(P_{k}\right.$ is a path on $k$ edges), then $\chi_{\mathcal{P}}^{\prime}\left(P_{n}\right)=n$ for every $n \geq 2$ while maximum degree is 2 .

\section{Generalized acyclic edge colorings :}

This notion was introduced in [5] and is a generalization of the acyclic edge colorings. For any $r \geq 3$, the $r$-acyclic chromatic index $a_{r}^{\prime}(G)$ is the minimum number colors sufficient to properly color the edges of $G$ so that every $k$-cycle uses at least $\min \{r, k\}$ colors, for every $k \geq 3$. Note that this specializes to the standard acyclic chromatic index when $r=3$. In [?], it is shown that for every fixed $r \geq 4, a_{r}^{\prime}(G)=O\left(d^{\lfloor r / 2\rfloor}\right)$.

This result follows as a corollary of Theorem 4.1. Let $l=\lfloor r / 2\rfloor+1$. Let $\mathcal{P}$ be defined by

$$
\mathcal{P}=\left\{\left(2, P_{3}\right),\left(3, P_{4}\right), \ldots,\left(l-1, P_{l}\right),\left(r-1,\left\{C_{k}: k>r\right\}\right)\right\} .
$$

Here, $P_{k}$ denotes a path on $k$ edges and $C_{k}$ denotes a cycle on $k$ edges. The first $l-2$ pairs forbid any path having $k \leq l$ edges being colored with fewer than $k$ colors. This, in turn, implies that any cycle $C_{k}$ on $k \leq r$ edges is colored with $k$ colors. The last pair takes care of the remaining cycles. Thus, every $\mathcal{P}$-edge coloring is also a generalized $r$-acylic edge coloring. It is easy to see that

$$
\begin{gathered}
\forall k, 3 \leq k \leq l, \theta\left(k-1, P_{k}\right)=k-1 \leq\lfloor r / 2\rfloor, \\
\theta\left(r-1,\left\{C_{k}: k>r\right\}\right)=\max _{k \geq 1} \frac{r+k-2}{k+1}=\frac{r-1}{2} \leq\lfloor r / 2\rfloor .
\end{gathered}
$$

Applying Theorem 4.1, for each fixed $r \geq 3$, we have $a_{r}^{\prime}(G) \leq \chi_{\mathcal{P}}^{\prime}(G)=$ $O\left(d^{\lfloor r / 2\rfloor}\right)$. The upper bound is tight upto a constant factor as shown in [?]. 
Note that if, instead of defining $\mathcal{P}$ as above, we had used the natural definition of

$$
\mathcal{P}=\left\{\left(2, C_{3}\right),\left(3, C_{4}\right), \ldots,\left(r-1,\left\{C_{k}: k \geq r\right\}\right)\right\},
$$

we would have only obtained a bound of $O\left(d^{r-2}\right)$. In fact, our choice of $\mathcal{P}$ was motivated by the choice of bad events used in [?]. This shows that it will help to try to upper bound a more restrictive coloring. We formally state and apply this observation in the following subsection.

\section{Improving some of the table entries}

For a connected graph $H$, let $d l(H)$ denote the diameter of the line graph of $H$. This means that any two edges in $H$ are part of a path in $H$ on at most $d l(H)+1$ edges. Note that if an edge coloring (proper or free) of $G$ is such that any path in $G$ on $k$ (for each $k \leq d l(H)+1$ ) edges uses exactly $k$ colors, then any copy of $H$ in $G$ must use at least $|E(H)|$ colors. Otherwise, there must be two edges in a copy of $H$ colored the same and since these are part of some path on $k \leq d l(H)+1$ edges, this path must use at most $k-1$ colors, a contradiction. This, in turn, implies that for any $j<|E(H)|$, any $j$ color classes of this coloring does not have a copy of $H$. This is a more restricted coloring than forbidding a copy of $H$ in any $j$ color classes. But, this may result in a better bound. By applying Theorem 4.1 to this observation, we get the following refinement of Theorem 1.1 whose proof can be easily worked out.

Theorem 5.1 Let $\mathcal{F}$ be a fixed family of connected graphs and let $j$ be a positive integer such that $j<\min _{H \in \mathcal{F}}(|E(H)|)$. Let $\mathcal{F}=\mathcal{F}_{1} \cup \mathcal{F}_{2}$ be a fixed partition of $\mathcal{F}$ where $\mathcal{F}_{1}$ is finite. Let $\theta_{2}=\theta\left(j, \mathcal{F}_{2}\right)$ and $\theta_{1}=$ $\max _{H \in \mathcal{F}_{1}} \min (d l(H), \theta(j,\{H\}))$ where $d l(H)$ is the diameter of the line graph of $H$. Then, there exists a constant $C=C\left(j, \mathcal{F}_{1}, \mathcal{F}_{2}\right)$ such that for any graph $G$ of maximum degree $d$, we have

(i) $\chi_{j, \mathcal{F}}^{\prime}(G) \leq C d^{\max \left(1, \theta_{1}, \theta_{2}\right)}$;

(ii) $f \chi_{j, \mathcal{F}}^{\prime}(G) \leq C d^{\max \left(\theta_{1}, \theta_{2}\right)}$;

The motivation for this theorem is that for a suitable choice of the partition $\mathcal{F}=\mathcal{F}_{1} \cup \mathcal{F}_{2}$, it may be that $\max \left\{\theta_{1}, \theta_{2}\right\}<\theta(j, \mathcal{F})$ resulting in an asymptotic improvement of the bound. This is illustrated in the following two improvements on entries in Table 1 in the previous section. 
1. For the planarity restriction with $j=8$, we can improve the upper bound to $O\left(d^{2}\right)$ from the $O\left(d^{4}\right)$ presented before. Write $\mathcal{F}=\mathcal{F}_{1} \cup$ $\mathcal{F}_{2}$ where $\mathcal{F}_{1}$ is the set of all subdivisions of $K_{3,3}$ with at most one subdivision and $\mathcal{F}_{2}=\mathcal{F} \backslash \mathcal{F}_{2}$. $\mathcal{F}_{1}$ has exactly two members and for each of them, the diameter of the corresponding line graph $L(H)$ is 2 and hence $\theta_{1}=2$. Also

$$
\theta\left(8, \mathcal{F}_{2}\right)=S U P_{k \geq 0, l \geq 2}\left\{\frac{3+k}{10-8+k}, \frac{4+l}{9-8+l}\right\}=2 .
$$

Thus, by Theorem 5.1, we can properly color the edges of a graph of maximum degree $d$ using $O\left(d^{2}\right)$ colors so that the union of any 8 color classes is planar.

2. For the outerplanarity restriction with $j=5$, write $\mathcal{F}=\mathcal{F}_{1} \cup \mathcal{F}_{2}$ where $\mathcal{F}_{1}$ is the set of all subdivisions of $K_{2,3}$ with at most one subdivision and $\mathcal{F}_{2}=\mathcal{F} \backslash \mathcal{F}_{1}$. For each of the two members in $\mathcal{F}_{1}$, the diameter of the corresponding line graph $L(H)$ is 2 and hence $\theta_{1}=2$. Also

$$
\theta\left(5, \mathcal{F}_{2}\right)=S U P_{k \geq 0, l \geq 2}\left\{\frac{2+k}{6-5+k}, \frac{3+l}{6-5+l}\right\}=2 .
$$

Thus, by Theorem 5.1, we can properly color the edges of a graph of maximum $d$ using $O\left(d^{2}\right)$ colors so that the union of any 5 color classes is outerplanar.

3. If we take $\mathcal{F}=\left\{K_{l}\right\}(l \geq 5)$ and set $j=\left(\begin{array}{l}l \\ 2\end{array}\right)-1$, then $\theta(j, \mathcal{F})=l-2 \geq 3$, $d l\left(K_{l}\right)=2, \mathcal{F}_{2}=\emptyset$ and $\theta_{1}=2$. Theorem 1.1, on the other hand, only provides a bound of $O\left(d^{l-2}\right)$ since $\theta\left(j, K_{l}\right)=l-2$.

The example 3 given above motivates the following special case of Theorem 5.1 which provides an improvement of Theorem 1.1 for finite families $\mathcal{F}$. It is explicitly stated below for the sake of completion.

Theorem 5.2 Let $\mathcal{F}$ be a finite family of connected graphs and let $j$ be a positive integer such that $j<\min _{H \in \mathcal{F}}|E(H)|$. Let $\theta_{1}=\theta_{1}(j, \mathcal{F})$ be defined as

$$
\theta_{1}(j, \mathcal{F})=\max \{\min (d l(H), \theta(j,\{H\}): H \in \mathcal{F}\} .
$$

Then, there exists a constant $C=C(j, \mathcal{F})$ such that for any graph $G$ of maximum degree $d$, we have

(i) $\chi_{j, \mathcal{F}}^{\prime}(G) \leq C d^{\max \left(1, \theta_{1}\right)}$.

(ii) $f \chi_{j, \mathcal{F}}^{\prime}(G) \leq C d^{\theta_{1}}$. 


\section{Another strengthening and list analogues}

We can further strengthen the asymptotic behavior of the upper bounds (as a power of $d$ ) on optimal free colorings in some cases. Given a pair $(j, \mathcal{F})$ with usual meanings, define $K(H)$, for each $H \in \mathcal{F}$, as any connected induced subgraph $K$ of $H$ with $|E(K)|>j$ and having the least possible value of $\frac{|V(K)|-2}{|E(K)|-j}$. Define $\mathcal{F}^{\prime}=\{K(H): H \in \mathcal{F}\}$. Define $\theta_{S}(j, \mathcal{F})=\theta\left(j, \mathcal{F}^{\prime}\right)$.

Then, any $\left(j, \mathcal{F}^{\prime}\right)$ edge coloring (proper or free) is also a $(j, \mathcal{F})$ edge coloring (proper or free). Also, $\theta\left(j, \mathcal{F}^{\prime}\right) \leq \theta(j, \mathcal{F})$ and the inequality can be strict possibly. As a result, one can in fact substitute $\theta_{S}(j, \mathcal{F})$ in place of $\theta(j, \mathcal{F})$ in Proposition 2.2 and Proposition 2.6.

However, it is easily verified that

$$
\frac{|V(K(H))|-2}{|E(K(H))|-j}<\frac{|V(H)|-2}{|E(H)|-j} \text { only if } \frac{|V(H)|-2}{|E(H)|-j}<1 .
$$

Hence, the possibility of an asymptotic improvement by using $\theta_{S}(j, \mathcal{F})$ is ruled out for proper $(j, \mathcal{F})$ chromatic indices. However, the asymptotic improvement is possible for upper bounds on free $(j, \mathcal{F})$ chromatic indices. For example, consider the graph $F$ on $[5]=\{1, \ldots, 5\}$ where the subset [4] induces a $K_{4}$ and 5 is adjacent to only 4 . Then $\theta(2,\{F\})=3 / 5$ but $\theta_{S}(2,\{F\})=1 / 2$. Thus, using $\theta_{S}(j, \mathcal{F})$ (in place of $\theta(j, \mathcal{F})$ ) allows us to get an improved bound of $O\left(d^{1 / 2}\right)$. Also, this strengthening can be extended to colorings forbidding several pairs of $(j, \mathcal{F})$ simultaneously.

The strengthening of Theorem 5.1 is not always achieved by the strengthening outlined above. It was noticed in Section 4 that Theorem 5.1 achieves asymptotically the bound of $O\left(d^{2}\right)$ on $\chi_{j, K_{l}}(G)$ for $j=\left(\begin{array}{l}l \\ 2\end{array}\right)-1$. But this bound is not achieved by the strengthening of this section, since $\mathcal{F}^{\prime}=\left\{K_{l}\right\}$.

List analogues : It can be verified that our proofs (based on probabilistic arguments) can be in fact easily be adapted to work for the list analogues of the $(j, \mathcal{F})$ edge colorings and chromatic indices. In the list version, each edge is given a list of colors and we are interested in determining the minimum size of any list which guarantees (irrespective of the actual contents of the lists) the existence of a $(j, \mathcal{F})$ edge coloring of $G$. We refer to the minimum size as the list $(j, \mathcal{F})$ chromatic index of $G$ (or the list $\mathcal{P}$ chromatic index of $G)$. Hence it follows that each of the Propositions 2.2 and 2.6 and Theorems 
4.1, 5.1 and 5.2 holds true even if we replace the chromatic index by its list analogue in the statement.

\section{Conclusions and Open Problems}

We considered a generalization of some known edge colorings like acyclic edge colorings and obtained upper bounds on the chromatic index in terms of the maximum degree $d$. We have not tried to optimize the constants mentioned in the statements and it is very likely that the constants can be brought down further to small values.

For several $(j, \mathcal{F})$ edge colorings, the bounds are actually $O(d)$, thereby showing that imposing additional restrictions involving any few color classes does not necessarily increase the required number of colors asymptotically. Obviously, these bounds are tight within a constant factor for such colorings. It would be interesting to establish the tightness (at least within constant or polylog multiplicative factor) of other super linear upper bounds.

It would also be interesting to obtain constructive (that is, deterministically and algorithmically efficiently realizable) bounds which match the bounds presented in this paper for some specific pairs $(j, \mathcal{F})$. For some colorings, there is an asymptotic gap between existential and deterministically constructible bounds. For example, acyclic chromatic index of any graph is at most $16 d$ but the currently known deterministically constructible bound (see [?]) is only shown to be $O(d \log d)$.

However, the recent breakthrough result of Moser and Tardos [?] on a constructive version of Lovász Local Lemma can be applied to the proof arguments of Theorem 1.1 resulting in a randomized algorithm with a polynomial expected running time for obtaining a $(j, \mathcal{F})$ edge coloring matching the upper bound. The details will appear elsewhere.

An interesting direction is to explore improvements in the bounds for random graphs or for random regular graphs. Such results have been obtained for acyclic edge coloring in [8] where it was shown that the acyclic chromatic index of a random $d$-regular graph is at most $d+1$ with high probability.

Acknowledgements : We thank an anonymous reviewer whose comments prompted us to include the remark after Corollary 4.2. 


\section{References}

[1] Noga Alon, Colin McDiarmid, and Bruce A. Reed. Acyclic coloring of graphs. Random Struct. Algorithms, 2(3):277-288, 1991.

[2] Noga Alon and Joel Spencer. The Probablistic Method. Wiley, 1992.

[3] Matt DeVos, Guoli Ding, Bogdan Oporowski, Daniel P. Sanders, Bruce A. Reed, Paul D. Seymour, and Dirk Vertigan. Excluding any graph as a minor allows a low tree-width 2-coloring. J. Comb. Theory, Ser. B, 91(1):25-41, 2004.

[4] D.West. Introduction to Graph Theory. Prentice-Hall, Inc., 2001.

[5] Stefanie Gerke, Catherine S. Greenhill, and Nicholas C. Wormald. The generalized acyclic edge chromatic number of random regular graphs. Journal of Graph Theory, 53(2):101-125, 2006.

[6] L.Loväsz, J.Pelikan, and K.Vesztergombi. Discrete Mathematics : Elementary and Beyond. Springer-Verlag, 2003.

[7] Jaroslav Nesetril and Patrice Ossona de Mendez. Tree-depth, subgraph coloring and homomorphism bounds. Eur. J. Comb., 27(6):1022-1041, 2006 .

[8] Jaroslav Nesetril and Nicholas C. Wormald. The acyclic edge chromatic number of a random $d$-regular graph is $d+1$. Journal of Graph Theory, 49(1):69-74, 2005.

[9] N.R.Aravind and C.R.Subramanian. Bounds on proper colorings with restrictions on the union of color classes. Submitted to Journal of Graph Theory, 2008. 